\title{
Euglena International Network (EIN): Driving euglenoids into the biotechnology world
}

ThankGod E. Ebenezer ${ }^{1 *}$, Ross S. Low ${ }^{2}$, Ellis Charles O'Neill ${ }^{3}$, I-Shuo Huang ${ }^{4}$, Antonio DeSimone $^{5}$, Scott C. Farrow ${ }^{6,7}$, Robert Andrew Field ${ }^{8}$, Michael Ginger ${ }^{9}$, Sergio Adrián Guerrero $^{10}$, Michael Hammond ${ }^{11}$, Vladimír Hampl ${ }^{12}$, Geoff Horst ${ }^{13}$, Takahiro Ishikawa ${ }^{14}$, Anna Karnkowska ${ }^{15}$, Eric W. Linton ${ }^{16}$, Peter Myler ${ }^{17}$, Masami Nakazawa ${ }^{18}$, Cardol Pierre ${ }^{19}$, Rosina Sánchez-Thomas ${ }^{20}$, Barry J Saville ${ }^{21}$, Mahfuzur R Shah ${ }^{6}$, Alastair G. B. Simpson ${ }^{22}$, Aakash Sur' ${ }^{17}$, Kengo Suzuki' ${ }^{23}$, Kevin M. Tyler ${ }^{24,25}$, Paul Zimba ${ }^{26}$, Neil Hall2,27, Mark C. Field ${ }^{11,28}$

${ }^{1}$ European Molecular Biology Laboratory, European Bioinformatics Institute, Wellcome Genome Campus, Hinxton, Cambridge CB10 1SD, UK, ${ }^{2}$ Earlham Institute, Norwich Research Park, Norwich NR4 7UZ, UK, ${ }^{3}$ University of Nottingham, Nottingham NG7 2RD, UK, ${ }^{4}$ Office of Regulatory Science, United States Food and Drug Administration, Center for Food Safety and Applied Nutrition, College Park, 20740, USA, ${ }^{5}$ The BioRobotics Institute, Scuola Superiore Sant'Anna, Pisa 56127, Italy, ${ }^{6}$ Discovery Biology, Noblegen Inc., Peterborough, Ontario K9L 1 Z8 Canada, ${ }^{7}$ Environmental and Life Sciences Graduate Program, Trent University, Peterborough, Ontario K9L 0G2 Canada, ${ }^{8}$ University of Manchester, Manchester M1 7DN, United Kingdom, ${ }^{9}$ School of Applied Sciences, University of Huddersfield, Huddersfield HD1 3DH, UK, ${ }^{10}$ Instituto de Agrobiotecnología del Litoral. CCT CONICET Santa Fe, Santa Fe 3000, Argentina, ${ }^{11}$ Institute of Parasitology, Biology Centre, Czech Acadamy of Sciences, České Budějovice 370 05, Czech Republic, ${ }^{12}$ Charles University, Faculty of Science, Department of Parasitology, BIOCEV, Vestec 25250, Czech Republic, ${ }^{13}$ Kemin Industries, Plymouth, Michigan, 48170 USA, ${ }^{14}$ Institute of Agricultural and Life Sciences, Academic Assembly, Shimane University, Matsue 690-8504, Japan, ${ }^{15}$ Institute of Evolutionary Biology, Faculty of Biology, University of Warsaw, Warsaw 02-089, Poland, ${ }^{16}$ Department of Biology, Central Michigan University, Mt. Pleasant, MI 48859 USA, ${ }^{17}$ Biomedical and Health Informatics, School of Medicine, University of Washington, WA 98195 USA, ${ }^{18}$ Osaka Prefecture University, Sakai 599-8531, Japan, ${ }^{19}$ Université de Liège, Liège, 4000, Belgium, ${ }^{20}$ Instituto Nacional de Cardiología, Ignacio Chávez, Mexico 14080 Mexico, ${ }^{21}$ Forensic Science, Environmental and Life Sciences, Trent University, Peterborough K9L 0G2, Canada, ${ }^{22}$ Department of Biology and Institute for Comparative Genomics, Dalhousie University, Halifax, Nova Scotia, Canada, ${ }^{23}$ Euglena Co. Ltd., Tokyo, Japan, ${ }^{24}$ University of East Anglia, School of Biological Sciences, Norwich NR4 7TJ, UK, ${ }^{25}$ Department of Biological Sciences, Faculty of Science, King Abdulaziz University, Jeddah, 21589, Saudi Arabia, ${ }^{26}$ PVZimba, LLC, 12241 Percival St, Chester 23831, USA, ${ }^{27}$ School of Biological Sciences, University of East Anglia, Norwich, Norfolk, U.K, ${ }^{28}$ School of Life Sciences, University of Dundee, Dundee, DD1 5EH, UK

*Corresponding author: thankgod1980@yahoo.co.uk 


\section{Abstract teaser}

Euglenoids show great promise to benefit our world; as biofuels, environmental remediators, anti-cancer agents, robotics design simulators and food nutritional agents, but the absence of reference genomes currently limit realizing these benefits. The Euglena International Network (EIN) (https:/leuglenanetwork.org/) aims to address these challenges, and is currently seeking formative phase support and funding.

\section{Body start}

Of the nearly 1000 known species of euglenoids (Triemer and Zakryś, 2015), including Euglena gracilis and Rhabdomonas costata, fewer than $2 \%$ have been explored for any level of translational potential in the past 20 years. The absence of reference genomes currently limits biotechnology applications, including the development of efficient tools for genetic manipulation in euglenoids.

EIN aims to advance euglenoid science through a creative amalgam of academic institutions, national research institutes and biotechnology industry, to translate and exploit euglenoids through genome sequencing. EIN has defined goals, mobilized scientists, established a clear roadmap (Grand Challenges), connected academic and industry professionals and is currently formulating policy and partnership principles, driven by EIN Executive and Science committees. However, for EIN's activities to be maintained and durable, long-term support is vital. We call on national and continental funding agencies and research councils, protists and algae communities, and biotechnology and pharmaceutical industries, to embrace, support and fund translational exploitation of these highly valuable organisms.

\section{The challenges}

It is rare to find a versatile organism that possess diverse roles in food, nutrition, nutraceuticals, ecological remediation and restoration, biofuels, social gaming, robotic engineering, biocomputing, anti-cancer drugs development, pharmaceuticals, animal health, $\mathrm{CO}_{2}$ sequestration, cosmetics, understanding microgravity and offer a potential source of calories and nutrients for extraterrestrial exploration (Table 1). The euglenoids naturally possess these biotechnological applications within a non-genetically modified system. For example, without genetic manipulations, euglenoids produce dozens of molecules including vitamins (e.g. A, B, C, D and E), polysaccharides (e.g. paramylon), lipids (e.g. saturated, monounsaturated and polyunsaturated fatty acids), and bioactive natural products like euglenophycin (Cabang, et al., 2017, Gissibl, et al., 2019). This differentiates euglenoids from many other biotechnology chassis such as bacteria and fungi where extensive modifications are required to produce metabolic precursors of sufficient value. For example, beta-glucans found in yeast are bound to the yeast cell wall making it expensive to modify (e.g. to obtain up to $50 \%$ beta-glucan) whereas in 
euglenoids beta-glucans are freely found and are not bound to a cell wall (Euglena does not possess a cell wall), making it less expensive to modify to obtain more than $90 \%$ beta-glucans. Similarly, in the face of climate change, euglenoids have demonstrated to be of relevance by absorbing high concentration of $\mathrm{CO}_{2}$ emissions, thereby reducing possible carbon footprints due to their high photosynthetic capabilities.

The euglenoids (Euglenida) are unicellular flagellates with an exceptionally wide geographical and ecological distribution including aquatic, and terrestrial ecological niches (Leander, et al., 2017). Euglenoids, in large part, owe their surprisingly diverse genetic repertoire to secondary endosymbiosis with a green alga (Dorrell, et al., 2017), exemplifying the genetic diversity in E. gracilis and other euglenoids. Euglena gracilis (Figure $1 \mathrm{~A}$ ) remains the most characterized euglenoid, due to a combination of interests as a model organism since the 1970s, taxonomic position, societal benefits, and significant biotechnological potential. A large genome of up to $3 \mathrm{~Gb}$, with nearly 40,000 protein coding genes, together with additional complexity due to alternate splicing, potentially underpins the wealth of natural products produced (Ebenezer, et al., 2019). These combined basic science and translational aspects of euglenoids can be maximized and fully explored, but are limited by the primitive nature of current genetic tools (Harada, et al., 2020). The development of genetic tools for euglenoids is hampered by lack of a high quality reference genome for any species of euglenoid - this has remained a challenge due to overall size and a considerable frequency of repetitive sequence.

\section{Why a network?}

The Euglena International Network (EIN) aims to harness the power of euglenoids science, exploiting the huge and unique biotechnology potential that euglenoids offer (Box 1). EIN kicked off in November 2020, and since its inception EIN has focused on formulating a partnership framework, scoping the Euglenoid Genomes Project (EGP), and establishing policy directives for EIN governance. For instance, EIN has connected with prospective industry partners e.g Euglena Co. Ltd. (https://www.euglena.jp), Kemin Industries (https://www.kemin.com), and Noblegen Inc (https://www.noblegen.com), as well as not-for-profit research centers and initiatives such as the Earlham Institute (EI), Manchester Institute of Biotechnology (MIB), Darwin Tree of Life (DToL), and the European Bioinformatics Institute (e.g. Ensembl Protist, UniProt) (Figure 1B). EIN plans to bring together 500 scientists, industry professionals, stakeholders, and partners, to supercharge euglenoid research, initially focusing on sequencing euglenoids genomes that span the breadth of euglenoid diversity and translating the data generated into societal benefits. 


\section{Why the EIN goals matter}

EIN plans to generate high quality reference genomes of the nearly 1000 euglenoids species in 10 years which will guide four critical future directions for euglenoid science and technology development:

1. Exploration, translation and commercialisation of euglenoid products: The key bottleneck in product development (e.g. food, nutrition, nutraceuticals) is the availability of high-value bioactive ingredients, molecules, and nutritional components capable of long-term potency. Euglenoids will solve industrial bioactive ingredient-based problems in the global biotechnology industry, with a market size of USD 177.7 billion in 2019 and expected to reach USD 298.54 billion by 2027. For instance, Euglena Co. Ltd. has commercialized human superfoods from Euglena gracilis, supplied biodiesel refined from Euglena wax esters, and aims to produce the first certified algae jet fuel blend (Table 1). Similarly, Kemin Industries has economically produced Euglena gracilis biomass rich in the immune modulating glycopolymer paramylon (beta-1,3-glucan) with clinical trials suggesting human immune, gastrointestinal, and even mental health and sleep benefits (Table 1). Finally, Noblegen Inc. is leveraging Euglena for water filtration and consumer facing foods (Eunite Foods), including Euglena protein-rich flour and Euglena meat analogues (e.g. chicken nuggets, pulled pork, smokey tuna) (Table 1).

To date, the translation and exploitation of euglenoids has focused on one species, Euglena gracilis. To maximize the full suite of euglenoids potential and advance market acceptance for the nearly 1000 euglenoids species, the translation and commercialisation of euglenoids must be accelerated, including scaling and supporting crowdfunding initiatives to carry out basic toxicological studies on generic strains of euglenoids crucial in easing regulatory hurdles. For example, this can be seen in the expensive, multi-year, and rigorous evaluation of safety data on BetaVia Complete during the Kemin European Food Safety Agency (EFSA) novel food permit for whole-cell Euglena gracilis (Table 1).

2. Maximize euglenoids applications in Ecological and Environmental management: One of the key bottlenecks in the cleanup of contaminated and polluted environments (i.e. bioremediation market) using living systems is the availability of efficient biological systems able to sequester pollutants without bioaccumulating along the food web or food chain (Moreno-Sánchez, et al., 2017). For example, the presence of euglenoids, and the ichthyotoxin euglenophycin produced by $E$. sanguinea (Zimba, et al., 2017), can act as indicators of changes in the environment. However, the drawback here is that $E$. sanguinea is lacking a reference genome to inform controlling conditions for environmental management 
and biomonitoring. To maximize potentials for biomonitoring, EIN will include several transcriptome environmental time points (e.g. high temperatures, low temperatures, light and dark conditions) as part of the EGP to fully capture expressed genes at different environmental and laboratory controlled conditions.

3. Understand the basic biology of euglenoids: Ploidy, DNA recombination preferences and the sexual habits of $E$. gracilis remain uncrowned but are critical as a prelude to sophisticated, robust forward or reverse genetics. For example, the current draft genome of $E$. gracilis suggests presence of a polyploidy genome configuration (Ebenezer, et al., 2019) albeit with some studies suggesting a haploid state (Ebenezer, et al., 2017). Molecular reconstruction of the evolutionary histories of euglenoid metabolism, gene regulation and how these protists perceive environmental cues will provide the fundamental basis for acting on opportunities to further commercialize euglenoid biology or develop euglenoid biosensors. EIN will develop robust forward / reverse genetics for one or more euglenoids species.

4. Understand the Evolution of euglenoids: The euglenoids are closely related to the kinetoplastids (e.g Trypanosomes and Leishmania) on the evolutionary tree of life. The kinetoplastids are parasites of humans, and other mammals, which causes immeasurable suffering and commercial loss across much of the globe. The study of euglenoids will help to expedite drug discovery and development against parasitic diseases such as trypanosomiasis (sleeping sickness) and leishmaniasis. For instance, mitochondrial investigations into Trypanosoma brucei have unearthed variety of diverged core structures including respiratory subunits (OXCT2 and NDUFS3) (Pagliarini, et al., 2008), protein import machinery (ATOM40, pATOM36 and TIM22) (Schneider 2018) and a mitochondrial contact site and cristae organization system (subunits Mic20 and Mic34) (Kaurov et al. 2018), all of which represent exciting medicinal candidates due to their importance for organism viability as well potentially being lineage-specific. To establish a more comprehensive collection of Euglenozoa knowledge for future therapeutic analysis, EIN will identify several basal euglenoid species and establish their cultivation for high-throughput genome sequencing, with particular focus on heterotrophic representatives of varied nutritional modalities.

\section{A call to action}

The production of high quality reference genomes of euglenoids will require a combination of short and long reads as well as the utility of high quality genome sequencing platforms with $>99 \%$ read accuracy such as the current PacBio Hi-Fi technology (Wenger, et al., 2019). We estimate that it will cost up to $\$ 10$ million USD to sequence 1000 euglenoids genomes over the next 10 years using a combination of long read technologies (e.g. PacBio Hi-HiFi), short reads (e.g Hi-C) and optical genome mapping (e.g. Bionano) as has been demonstrated by the Vertebrate Genomes Project (VGP) (Rhie, et al., 2021). 
This estimated cost also reflects any actual, or in-kind, contributions from larger genome consortia and partners to support the EGP goals. We call on national and regional funding agencies, protists and algae scientific communities (e.g. International Society for Protistology, ISP; International Phycological Society, IPS; World Federation of Parasitologists, WFP), biotechnological and pharmaceutical companies, to embrace euglenoids as unique and valuable organisms for their basic sciences and translational processes, and provide formative phase funding to support the objectives of EIN.

\section{Next steps}

EIN will make five bold steps to address the identified Grand Challenges in euglenoid science:

1. Formation of the Euglenoid Genomes Project (EGP) is key: To build the foundation required to deliver on EIN's promises, EIN will progress to initiate the Euglenoid Genomes Project (EGP) with the goal of exploration and exploitation of accurate, or new generation, technologies (e.g. a combination of $\mathrm{PacBio} \mathrm{Hi}-\mathrm{Fi}, \mathrm{Hi}-\mathrm{C}$, and Bionano) to produce 1000 reference genomes of euglenoids. These technologies will help to solve the problems of fragmented nature of euglenoids genomes experienced in E. gracilis (Ebenezer, et al., 2019) by providing both short reads, optical maps, and chromosomal-sized reads.

2. Unlock euglenoids potential through an Open EIN: To maximize scientific contributions and benefits, EIN will adhere to the FAIR principle (findable, accessible, interoperable, and reusable) by publishing and depositing all genomic data in open access journals and public databases such as the International Nucleotide Sequence Database Collaboration (INSDC) and Ensembl Protist respectively. For instance, as part of EGP, EIN plans to set up the Euglenoids Genome Browser to allow open curation of the genomic data generated, including identifying metabolites, and contributions in generating secondary structures of euglenoids proteins through AlphaFold (Jumper, et al., 2021).

3. Bioprospecting of high value products, strains and species: E. gracilis produce a plethora of known valuable products (e.g. glycopolymers and small molecules) but we still lack a detailed library of products (i.e. metabolites, proteins, genes) within E. gracilis or euglenoids in general. These products, strains and species could be tremendously important for solving global challenges like disease prophylaxis, treatments, and combating poor nutrition. EIN will take advantage of modern analytical and omics tools to identify and characterize known euglenoid strains and species, including their natural product profiles and genetic or protein complements. 
4. Develop tools and techniques for genetic interrogation and modification: We believe that providing open access to EIN's genomic data will accelerate exploration of the plethora of uncharacterised euglenoid genes, a potential treasure trove for the emerging fields of biocatalysis and synthetic biology. For instance, reverse genetics techniques would allow access to the functions of uncharacterised euglenoid genes, and thus, their potential for commercial application and production of high-value compounds and therapeutics (e.g. maximizing the applications of euglenophycin in anti-cancer therapy).

5. Build a framework for commercial and academic collaborations: As biotech companies emerge and utilize euglenoids in their products, there will be increasing opportunities for academics to form synergies with industry. EIN will continue to serve as a platform for realizing potential academic and industry partnerships, whether it be to advance fundamental research or explore a commercial product that would benefit many interested parties. For example, researchers that identify and optimize the expression of a target molecule (e.g. tocopherol) could partner with a large-scale industry producer, and perhaps even a third partner, to market and sell the product.

Finally, EIN must be beneficial and open to communities in both developing and developed economies. Such benefits could be realized in the generation of Intellectual Property (IP), creation of jobs in new frontiers, production and administration of highly nutritious, but cost effective, euglenoids nutrients as well as improved bioeconomy. 


\section{Display items (Figure 1 and Table 1)}
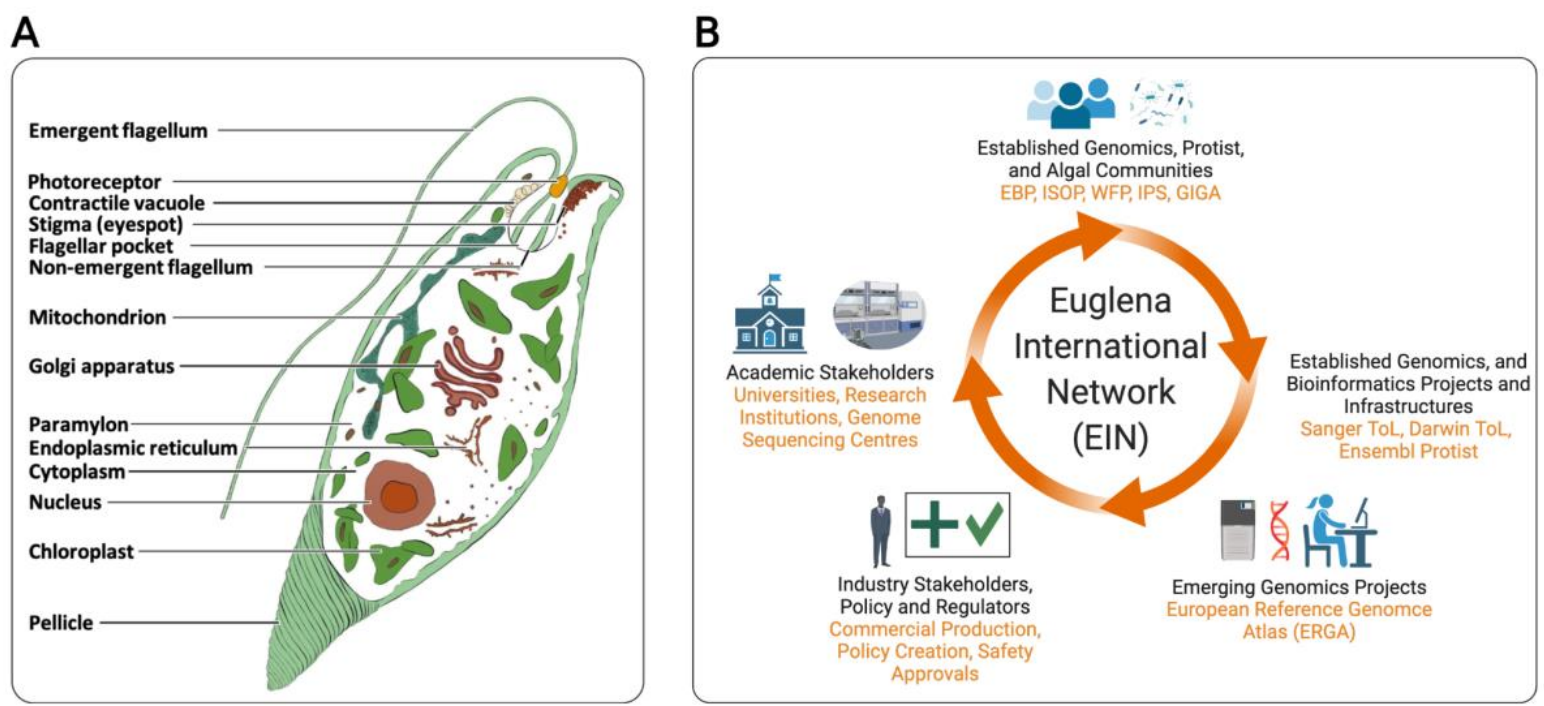

Figure 1: A. Euglenoids are single-cell organisms with a standard complement of internal cellular organelles for photosynthetic organisms with one to two emergent flagella. Obvious morphological features unique to euglenoids are the pellicle, a series of interlocking protein strips below the plasma membrane. Examples of metabolic features include paramylon, a $\beta-1,3$ glucan and the production of combustible wax esters. B. EIN aims to meet challenges and ambitions through multi-institutional, multicountry and multi-sector partnerships. Earth BioGenome Project (EBP), International Society for Protistology (ISOP), World Federation of Parasitology (WFP), International Phycological Society (IPS), Global Invertebrates Genomics Alliance, Sanger Tree of Life (Sanger ToL), Darwin Tree of Life (DToL) and European Reference Genome Atlas (ERGA) are all identified, prospective EIN partners. 
Table 1: Some diverse roles of euglenoids: Ongoing or past efforts

\begin{tabular}{|c|c|c|}
\hline $\mathbf{S} / \mathbf{N}$ & Roles & Web links \\
\hline 1 & $\begin{array}{l}\text { Food, nutrition and } \\
\text { nutraceuticals }\end{array}$ & $\begin{array}{l}\text { https://doi.org/10.2903/j.efsa.2020.6100 } \\
\text { https://eur- } \\
\text { lex.europa.eu/eli/reg impl/2020/1820/oj } \\
\text { https://doi.org/10.1016/j.algal.2018.11.024 }\end{array}$ \\
\hline 2 & $\begin{array}{l}\text { Ecological remediation and } \\
\text { restoration }\end{array}$ & $\begin{array}{l}\underline{\underline{\text { https://onlinelibrary.wiley.com/doi/10.1002/bit. }}} \\
\underline{\text { https://doi.org/10.3389/fbioe.2021.662655 }}\end{array}$ \\
\hline 3 & Biofuels & $\begin{array}{l}\underline{\text { https://doi.org/10.1186/s13068-019-1544-2 }} \\
\underline{\underline{\text { https://doi.org/10.1016/i.enconman.2016.08.0 }}} \\
\underline{\text { https://doi.org/10.3389/fbioe. } 2019.00108}\end{array}$ \\
\hline 4 & Social gaming & $\begin{array}{l}\text { https://doi.org/10.1371/journal.pone.0162602 } \\
\text { https://doi.org/10.1145/3313831.3376378 } \\
\text { https://www.nature.com/articles/s41587-019- } \\
\underline{0272-2} \\
\underline{\text { https://www.pnas.org/content/116/12/5411 }} \\
\underline{\text { https://www.nature.com/articles/s41567-018- }} \\
\underline{0277-7}\end{array}$ \\
\hline 5 & $\begin{array}{l}\text { Robotic engineering } \\
\text { (Soft robots) }\end{array}$ & $\frac{\text { https://ieeexplore.ieee.org/document/797637 }}{\underline{2}}$ \\
\hline 6 & Biocomputing & https://www.pnas.org/content/116/12/5411 \\
\hline 7 & Anti-cancer drug development & https://www.oncotarget.com/article/22238/ \\
\hline 8 & Pharmaceuticals & $\begin{array}{l}\underline{\text { https://www.ncbi.nlm.nih.gov/pmc/articles/PM }} \\
\underline{\text { C6213005/ }} \\
\underline{\text { https://onlinelibrary.wiley.com/doi/10.1111/ffd. }} \\
\underline{12871}\end{array}$ \\
\hline
\end{tabular}




\begin{tabular}{|l|l|l|}
\hline 9 & Animal health & $\underline{\text { https://doi.org/10.3382/ps/pey227 }}$ \\
\hline 10 & $\begin{array}{l}\text { CO2 Sequestration and } \\
\text { Fixation }\end{array}$ & $\begin{array}{l}\text { https://doi.org/10.1371/journal.pone.0162827 } \\
\text { https://pubmed.ncbi.n/m.nih.gov/16171688/ }\end{array}$ \\
\hline 11 & Cosmetics & $\begin{array}{l}\underline{\text { https://doi.org/10.3389/fbioe.2021.713840 }} \\
\text { https://doi.org/10.1292/ivms.09-0526 }\end{array}$ \\
\hline 12 & Understanding microgravity & $\underline{\text { htps://patents.google.com/patent/US874135 }}$ \\
\hline 13 & $\begin{array}{l}\text { Sources of calories and } \\
\text { nutrients for extraterrestrial } \\
\text { exploration }\end{array}$ & $\begin{array}{l}\underline{\text { https://doi.org/10.3389/fpls.2019.01621 }} \\
\underline{\text { https://www.nasa.gov/centers/ames/engineeri }}\end{array}$ \\
\hline 14 & $\begin{array}{l}\text { Additionally relevant link } \\
\text { https://doi.org/10.3389/fpls.2019.01621 }\end{array}$ \\
\hline
\end{tabular}




\section{References}

Cabang, A. B., De Mukhopadhyay, K., Meyers, S., Morris, J., Zimba, P. V., \& Wargovich, M. J. (2017). Therapeutic effects of the euglenoid ichthyotoxin, euglenophycin, in colon cancer. Oncotarget, 8(61), 104347-104358. https://doi.org/10.18632/oncotarget.22238

Dorrell, R. G., Gile, G., McCallum, G., Méheust, R., Bapteste, E. P., Klinger, C. M., ... Bowler, C. (2017a). Chimeric origins of ochrophytes and haptophytes revealed through an ancient plastid proteome. ELife, 6. https://doi.org/10.7554/eLife.23717 Ebenezer, T. E., Carrington, M., Lebert, M., Kelly, S., \& Field, M. C. (2017). Euglena gracilis Genome and Transcriptome: Organelles, Nuclear Genome Assembly Strategies and Initial Features. In Advances in experimental medicine and biology (Vol. 979, pp. 125-140). https://doi.org/10.1007/978-3-319-54910-1 7 Ebenezer, T. E., Zoltner, M., Burrell, A., Nenarokova, A., Novák Vanclová, A. M. G., Prasad, B., ... Field, M. C. (2019). Transcriptome, proteome and draft genome of Euglena gracilis. BMC Biology 2019 17:1, 17(1), 1-23. https://doi.org/10.1186/S12915-019-0626-8

Gissibl, A., Sun, A., Care, A., Nevalainen, H., \& Sunna, A. (2019). Bioproducts From Euglena gracilis: Synthesis and Applications. Frontiers in Bioengineering and Biotechnology, 7, 108. https://doi.org/10.3389/FBIOE.2019.00108

Harada, R., Nomura, T., Yamada, K., Mochida, K., \& Suzuki, K. (2020). Genetic Engineering Strategies for Euglena gracilis and Its Industrial Contribution to Sustainable Development Goals: A Review. Frontiers in Bioengineering and Biotechnology, 0, 790. https://doi.org/10.3389/FBIOE.2020.00790 Jumper, J., Evans, R., Pritzel, A., Green, T., Figurnov, M., Ronneberger, O., ... Hassabis, D. (2021). Highly accurate protein structure prediction with AlphaFold. Nature 2021 596:7873, 596(7873), 583-589. https://doi.org/10.1038/s41586-021$\underline{03819-2}$

Kaurov, I., Vancová, M., Schimanski, B., Cadena, L. R., Heller, J., Bílý, T., ... Hashimi, H. (2018). The Diverged Trypanosome MICOS Complex as a Hub for Mitochondrial Cristae Shaping and Protein Import. Current Biology, 28(21), 33933407.e5. https://doi.org/10.1016/J.CUB.2018.09.008

Leander, B. S., Lax, G., Karnkowska, A., Simpson, A. G. B. (2017). Euglenida. Handbook of the Protists, J.M. Archibald et al. (Eds.), Springer-Verlag, Wien. DOI https://doi.org/10.1007/978-3-319-32669-6 13-1

Moreno-Sánchez, R., Rodríguez-Enríquez, S., Jasso-Chávez, R., Saavedra, E., \& García-García, J. D. (2017). Biochemistry and Physiology of Heavy Metal Resistance and Accumulation in <Emphasis Type="Italic">Euglena </Emphasis $>$. Advances in Experimental Medicine and Biology, 979, 91-121. https://doi.org/10.1007/978-3-319-54910-1 6 
Pagliarini, D. J., Calvo, S. E., Chang, B., Sheth, S. A., Vafai, S. B., Ong, S.-E., ... Mootha, V. K. (2008). A Mitochondrial Protein Compendium Elucidates Complex I Disease Biology. Cell, 134(1), 112-123.

https://doi.org/10.1016/J.CELL.2008.06.016

Rhie, A., McCarthy, S. A., Fedrigo, O., Damas, J., Formenti, G., Koren, S., ... Jarvis, E. D. (2021). Towards complete and error-free genome assemblies of all vertebrate species. Nature 2021 592:7856, 592(7856), 737-746.

https://doi.org/10.1038/s41586-021-03451-0

Schneider, A. (2018). Mitochondrial protein import in trypanosomatids: Variations on a theme or fundamentally different? PLOS Pathogens, 14(11), e1007351. https://doi.org/10.1371/JOURNAL.PPAT.1007351

Triemer, R. E., \& Zakryś, B. (2015). Photosynthetic Euglenoids. In Freshwater Algae of North America: Ecology and Classification (pp. 459-483). https://doi.org/10.1016/B978-0-12-385876-4.00010-4

Wenger, A. M., Peluso, P., Rowell, W. J., Chang, P.-C., Hall, R. J., Concepcion, G. T., ... Hunkapiller, M. W. (2019). Accurate circular consensus long-read sequencing improves variant detection and assembly of a human genome. Nature Biotechnology 2019 37:10, 37(10), 1155-1162. https://doi.org/10.1038/s41587019-0217-9

Zimba, P. V., Huang, I.-S., Gutierrez, D., Shin, W., Bennett, M. S., \& Triemer, R. E. (2017). Euglenophycin is produced in at least six species of euglenoid algae and six of seven strains of Euglena sanguinea. Harmful Algae, 63, 79-84.

https://doi.org/10.1016/i.hal.2017.01.010 